\title{
Prediction of Extreme Response of Nonlinear Oscillators Subjected to Random Loading Using the Path Integral Solution Technique
}

\section{Arvid Naess}

Faculty of Civil Engineering, The Norwegian Institutc of Technology,

Rich. Birkelands vei la, N-7034 Trondheim, Norway
This paper studies the applicability of the path integral solution technique for estimating extreme response of nonlinear dynamic oscillators whose cquations of motion can be modclicd by the use of Irô stochastic differential equations. The state vector process associated with such a model is generally a diffusion process, and the probability density function of the state vector thus satisfies the FokkerPlanck-Kolmogorov equation. It is shown that the path integral solution lechnique combined with an appropriate numerical scheme constitutes a powerful mehod for solving the Fokker-Planck-Kolmogorow equation with natural boundary condi- tions. With the calculated probability density function of the state vector in hand. one can proceed to calculate the required quantities for estimating extreme response. The proposed method distinguishes itself by remarkably high accuracy and numerical robustness. These features are highlighted by application to example studies of nonlinear oscillators excited by white noise.

Key words: extreme response; nonlinear oscillators; path integral solution; raxdom loading.

Accepted: March 22, 1994

\section{Introduction}

An important element in the safety assessment of many engineering systems, is the task of estimating the probability of extreme events that may jeopardize the structure in some specificd sense. Very often, this problem can be formulated as finding the probability that some time varying random quantity does not exceed a specified capacity level during a given time period. Stated this way, the problem typically reduces to a study of the extreme values of a stochastic process originating as the response of a system subjected to some stochastic loading process.

In this paper the focus will be on the problem of estimating the extreme responsc of nonlinear dynamic systems subjected to random forcing processes. In recent years the methods of time domain Monte Carlo simulations, see, e.g., Refs. [1-5], have received consid- erable attention as a tool for estimating response statistics. These methods are versatile and attractive in the sense that nonlinearities can be easily dealt with. The main drawback at present is the largc CPU times needed for accuratc prediction of extremc responses. Even if this issue seems to become less of an obstacle every year, portending perhaps that such methods may dominate practical estimation of rcsponse statistics of nonlinear systerns in the not 100 distant future, it will still be desirable to have available alternative methods of calculating the rcsponse statistics, both simplified and more elaborate. Here we shall explore a method based on the theory of Markov diffusion processes. The justification for using this theory is related to the fact that the response of nonlinear dynamic systems to broad band random excitation can very often be accurately de- 
scribed by applying the theory of multidimensional Markov processes. By this, the extensive theory of Markov diffusion processes can be brought to bear on these problems. In particular, it can be shown that the probability law of response quantities can be derived by solving a partial differential cquation, viz.. the FokkerPlanck (-Kolmogorov) (FPK) equation, see Refs. [6,7]. In most cascs of practical interest, this cquation has to be solved numerically.

In the next section we shall describe a method for solving the FPK equation that is based on a formal solution of the same equation. This solution is obtained by invoking the fact that a Markov diffusion process locally looks like a Brownjan motion. By using the Markov property. the global solution can then be constructed by linking the local solutions, which are known explicitly. The obtained solution is generally known as a path integral solution (PIS). The rcader is referred to Ref. [7] for a further discussion. One of the first efforts to exploit the PIS method explicitly in developing numerical solution algorithms is described in Ref. [8]. Subsequently, other authors have also used the PIS approach to solve various random vibration problems, $\mathrm{cf}$. Refs. [9-13].

Before embarking on a description of the PIS method, it is expedient to briefly show how the obtained solutions are used in an extremc value analysis. Assuming that the response quantity of interest is a scalar (real) stationary stochastic process, $Z(t)$ say, the PIS method typically provides a numerical estimate of the joint probability density function (PDF) $f_{2}(\cdot *)$ of $Z(t)$ and $\dot{Z}(t)=\mathrm{d} Z(t)$ $\mathrm{dt}$. It is now assumed that the mean level upcrossing ratc $\nu_{7}^{*}(\cdot)$ of $Z(r)$ can he calculated from Rice's formula as follows

$$
\nu(z)=\int_{0}^{\infty} y f_{z z}(z, y) \mathrm{d} y .
$$

Adopting the assumption that upcrossing of high levels are statistically independent events, which leads to Poisson distributed crossings, it follows that an asymptotic approximation of the probability distribution function of the extreme value of the process $Z(t)$ during a time $T$, denoted by $M(T)$ ( $=\sup \{Z(t): 0 \leq f \leq T\})$, is given by

$$
\operatorname{Prob}\{M(T) \leq z\}-\operatorname{cxp}\left\{-\nu^{*}(z) T\right\} \quad(T \rightarrow \infty) .
$$

The accuracy of Eq. (2) depends to a large extent on the effective bandwidth of the response process $Z(t)$. Decreasing bandwidth leads cventually to a significant clumping $\mathrm{cffect}$ of large response pcaks, invalidating the assumption of statistically independent upcrossing of high levels. Mcthods that aim at correcting for this effect have been proposed for Gaussian (Refs. $[14,15]$ ) and
non-Gaussian (Ref. [16]) processes. However, this point will not be pursued any further here. We shall assume that $\mathrm{Eq}$. (2) provides an acceptable approximation. Hence, the central parameter to be determined is the upcrossing frequency $\nu_{(}^{*}\left({ }^{\circ}\right)$, which is casily calculated once the joint PDF $f(\bullet \cdot \bullet)$ of $Z(t)$ and $Z(t)=\mathrm{d} Z(t) / \mathrm{d} t$ has been made available. In the next scction it is shown how this PDF can be calculated for the response of a widc range of nonlinear oscillators subjected to white noise or filtered whitc noise loading.

\section{The Path Integral Solution}

The path integral solution (PIS) method is suitable for calculating the joint probability density function (PDF) of a vector process $X(t)=\left[X_{1}(t) \ldots X_{a}(t)\right]^{T}$ (T-transposit tion) satisfying a stochastic differential equation of the following form, cf. Ref. [6].

$$
\mathrm{d} X(t)=m[X(t)] \mathrm{d} t+Q[X(t)] \mathrm{d} W(t) .
$$

Here $m(\bullet)=\left[m_{l}(\bullet), \ldots, m_{n}(\cdot)\right]^{\tau}, m_{\lambda}(\bullet)$ denotes a real function of $n$ real variables. $Q(\cdot)=\left(q_{i j}(\bullet)\right)$ denotes an $n \times m$ matrix where each $q_{l j}(\cdot)$ is a real function of $n$ real variables. $\quad W(t)=\left[W_{1}(t), \ldots, W_{m}(t)\right]^{T}$ where $W_{j}(t)$, $j=1, \ldots, m$ are standard, real Brownian motim processes, which are mutually independent, scc c.g., Refs. [6,7], That is, $E\left[W_{j}(t)\right]=0$ and

$$
\left.\Leftrightarrow\left[\mathrm{d} W_{i}(\mathrm{t}) \mathrm{d} W_{j}(l+\tau)\right]=\delta_{\eta,} \delta_{r, t+} \mathrm{d} \tau, i, j=\right], \ldots, m .
$$

where $\delta_{x y}=1$ for $x=y, \delta_{x y}=0$ for $x \neq y$. Equation (4) is a short-hand notation for the relation $E\left[\iint h\left(s_{+}\right) \mathrm{d} W_{j}(s) \mathrm{d} W_{j}(t)\right]=\delta_{i j} \int h(t, t) \mathrm{d} t$, where $h(\bullet \bullet$ is a non-random function.

Equation (3) is interpreted here as an Itô stochastic differential equation (SDE). Since it is often relevant to consider Eq. (3) as being obtained as a limit of equations with band limited noise processes, it may happen that $\boldsymbol{m}(\cdot)$ should contain corrcction terms to ensure a consistent limiting solution, cf. Ref. [6]. It is assumed here that this consideration has already been made, and that Eq. (3) has the final form to be used subsequently.

It is dernonstrated in Ref. [6] that the solution $X(t)$ to Eq. (3) is a Markov vector process. Its transition probability density function (TPD), $p\left(x, t \mid \mathbf{x}^{t}, t^{\prime}\right)$, is defined by the equation

$$
\operatorname{Prob}\left\{X(t) \in A \mid X\left(t^{\prime}\right)=x^{\prime}\right\}=\int \cdots \iint p\left(x, t \mid \mathbf{x}^{\prime}, t^{\prime}\right) \mathrm{d} x,(5)
$$

where $A \subseteq \mathbf{R}^{n}$ is some event, $\boldsymbol{x}, \boldsymbol{x}^{\prime} \in \mathbf{R}^{n}, \mathrm{~d} \boldsymbol{x}=\mathrm{d} x_{1} \ldots \mathrm{d} x_{n}$. 
Provided that $m(\bullet)$ and $Q(\bullet)$ satisfy certain regularity conditions, see Rcf. [6], it can be proved that the TPD $p\left(x, t \mid x^{\prime}, t^{\prime}\right)\left(t \geq t^{\prime} \geq 0\right)$ is the solution of a partial differcntial equation of the form

$$
\begin{gathered}
\frac{\partial}{\partial t} p\left(x, t \mid x^{\prime}, t^{\prime}\right)=-\sum_{i=1}^{n} \frac{\partial}{\partial x_{i}}\left[m_{i}(x) p\left(x, t \mid x^{\prime}, t^{\prime}\right)\right] \\
+\frac{1}{2} \sum_{i=1}^{n} \sum_{j=1}^{n} \frac{\partial^{2}}{\partial x_{i} \partial x_{j}}\left[g_{i j}(x) p\left(x, t \mid x^{\prime}, t^{\prime}\right)\right]_{*}
\end{gathered}
$$

where $\quad \boldsymbol{G}(\boldsymbol{x})=\left(g_{i j}(\boldsymbol{x})\right)=Q(\boldsymbol{x}) \boldsymbol{Q}(\boldsymbol{x})^{T}=\left(\sum_{i-1}^{m} q_{d} q_{j k}\right)$, and with initial condition $p\left(x, f^{\prime} \mid x^{\prime}, t^{\prime}\right)=\delta\left(x-x^{\prime}\right) . G(\cdot)$ will be called the diffusion matrix and Eq. (6) will be referred to as the Fokker-Planck-Kolmogorov (FPK) equation. Since clcarly $\operatorname{Prob}\left\{\boldsymbol{X}(t) \in \mathbf{R}^{n} \mid \boldsymbol{X}\left(t^{\prime}\right)=\boldsymbol{X}^{\prime}\right\}=1$ the TPD satisfies the following normalization condition

$$
\int \ldots \int p\left(x, l \mid x^{\prime}, t^{\prime}\right) \mathrm{d} x=1 \text {. }
$$

Let $f(x, t)$ denote the PDF of the randont vector $X(t)$. If $f\left(x, l^{\prime}\right)=w(x)$ for some initial PDF $w(x)$, then it is recognized from Eq. (6) and the relation

$$
f(x, t)=\int \ldots \int p\left(x, t \mid x^{\prime}, t^{\prime}\right) w\left(x^{\prime}\right) \mathrm{d} x^{\prime}
$$

that $f(x, t)$ itself is a solution of Eq. (6) satisfying the initial condition $f\left(x, l^{\prime}\right)=w^{\prime}(x)$.

In this paper we shall be interested primurily in sta tionary solutions $f_{s}(\boldsymbol{x})$ to Eq. (6), that is

$$
f_{s}(\boldsymbol{x})=\lim _{t \rightarrow \infty} f(\boldsymbol{x}, t)=\lim _{l \rightarrow \infty} p\left(x, l \mid x^{\prime}, t^{\prime}\right)
$$

provided they exist. Even when both limits exist, it is clear that $\lim f(x, t)$ provides the faster convergence when the initial condition $f\left(\boldsymbol{x}, t^{\prime}\right) \approx f_{3}(\boldsymbol{x})$. This comment is relcvant to the numerical implementation of the PIS method, and will be discussed below.

To obtain the PIS appropriatc for the dynumic systems studied in this paper, it is necessary to be more specific on the structure of the matrix function $Q(\cdot)$. In particular, it will be assumed that the first $r$ rows of $Q(\bullet)$ are zcro, that is

$$
q_{i j}(\cdot)=0 \text { for } i=1, \ldots, r ; j=1, \ldots, m(r<n)
$$

and that $q_{i j}(\cdot) \neq 0$ for at least onc $j$ for every $i=r+1, \ldots, n$. This implies that the diffusion matrix $G(\bullet)$ assumes the form

$$
G(\cdot)=\left[\begin{array}{cc}
O & 0 \\
O & G(\cdot)
\end{array}\right]
$$

$O$ denote appropriate zcro-matrices and $\bar{G}(\bullet)$ denotes an $(n-r) \times(n-r)$-matrix function with elements $g_{i j}(\cdot)$, $i j=r+1, \ldots, n, \tilde{G}(\cdot)$ will be called the reduced diffusion matrix. Equation (6) can now be rewritten as

$$
\begin{aligned}
& \frac{\partial}{\partial t} p\left(x, t \mid x^{\prime}, t^{\prime}\right)=-\sum_{j=1}^{n} \frac{\partial}{\partial x_{i}}\left[m_{i}(x) p\left(x, t \mid x^{\prime}, t^{\prime}\right)\right] \\
& +\frac{1}{2} \sum_{j=r+1}^{n} \sum_{j, r+1}^{n} \frac{\partial^{2}}{\partial x_{i} \partial x_{j}}\left[g_{i j}(x) p\left(x, t \mid x^{s}, t^{\prime}\right)\right] .
\end{aligned}
$$

Proceeding in a marner similar to the derivations in Ref. [7], it can be shown that the TPD for small values of $\tau\left(=t-t^{\prime}\right)$ is given by the following expression, which is correct up to terms of order $\tau^{2}$

$$
\begin{gathered}
p\left(x, t+\tau \mid x^{s}, t\right)=\left\{\prod_{i=1}^{r} \delta\left(x_{i}-x_{i}^{\prime}-m_{i}\left(x^{\prime}\right) \tau\right)\right\} \\
\cdot(2 \pi \tau)^{-\frac{n}{2}}\left|\tilde{G}\left(x^{\prime}\right)\right|^{-\frac{1}{2}} \cdot \operatorname{cxp}\left\{-\frac{1}{2 \tau} \sum_{i=r+1}^{n} \sum_{j+r+1}^{n}\right. \\
\left.\left(x_{i}-x_{i}^{\prime}-m_{i}\left(x^{\prime}\right) \tau\right)\left[\tilde{G}\left(x^{\prime}\right)^{\prime}\right]_{i, j-r}\left(x_{j}-x_{j}^{\prime}-m_{j}\left(x^{\prime}\right) \tau\right)\right\},
\end{gathered}
$$

where $|\tilde{G}|$ denotes the determinant of the reduced diffusion matrix $\bar{G}$, assumed to be positive definite. This implies that $|\tilde{G}|>0,\left[\tilde{G}^{-}\right]_{i j}$ denotes the element in position $i j$ of the inverse matrix of $\tilde{G}$. As shown in Ref. [7], the expression given by Eq. (13) is not unique, but secrns to be well suited for cur purpose.

Having obtaincd an explicit expressiun for the TPD for a short time step, one can now invoke the Markov property. This allows a TPD ower a tims interval of arbitrary length to be expressed in terms of a product of short-time TPDs. By dividing a given time interval $\left(t^{t}, l\right)$ into $N$ small time intervals of length $\tau-\left(t-t^{\prime}\right) / N$, it is found that $\left(t_{i}=t^{\prime}+j \tau, t=t^{\prime}, x=x^{(A)}, t^{\prime}=t_{0}, x^{\prime}=x^{(0)}\right)$

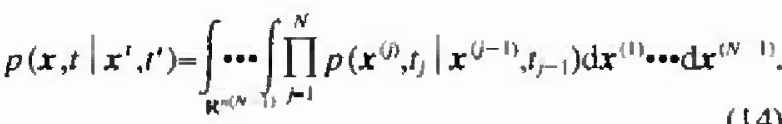

Similarly, with an in itial PDF $f\left(x^{\prime}, t^{\prime}\right)=w\left(x^{\prime}\right)$, the PDF $f(\boldsymbol{x}, t)$ will be given by

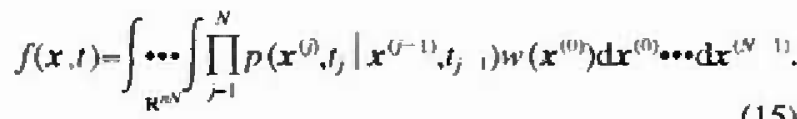

Hence, by combining Eq. (13) with Eqs. (14) or (15), a formal (approximate) solution of the FPK cquation can be written. Equations (14) and (15), which are often referred to as PIS, constitute the core of the numerical 
solution procedurc to be described subsequently. It is realized that a numerical solution according to this method, automatically provides the evolution in time of the (conditional) PDF of the Markov process $X(t)$ from given start conditions in terms of an initial density $f\left(x^{\prime}, t^{\prime}\right)=w\left(x^{\prime}\right)$, including the degenerate casc $f\left(x^{\prime}, t^{\prime}\right)-\delta\left(x^{\prime}-x_{0}\right)$, for some starting point $x_{0 \cdot}$. It is also worth noting how the PIS relates to the physics of the dynamic model, which is expressed through the coefficients $m_{j}(\bullet)$ and $q_{i j}(\bullet)$, cf. Eq. (3). The evolution in time of the PDF as expressed by the PIS, is scen to be directly determined by these coefficients in an explicit manner. This fact is a very important advantagc of the PIS method, and reveals its fundamental physical significance.

\section{Numerical Implementation}

In the numerical implementation, the PIS is obtained by an iteration process based on the Chapnan-Kolmogorov equation expressed as

$$
\begin{gathered}
p\left(x^{(j)}, t_{j} \mid x^{t}, t^{\prime}\right)=\int \cdots \int p\left(x^{(0,}, t_{j} \mid x^{(j-1)}, t_{j-1}\right) p\left(x^{(j-1)},\right. \\
\left.t_{j-1} \mid x^{\prime}, t^{\prime}\right) \mathrm{d} x^{(j-1)} .
\end{gathered}
$$

The discretization of state space for the numerical solution makes it appropriate to employ an interpolation and smoothing procedure to increase the numerical efficiency. It was found that application of cubic B-splines, as detailed in Ref. [17], offered the desired accuracy and smoothness for the type of problems considered in this paper. This procedure was used as follows. At each time step $t_{j-1} \rightarrow t_{j}, p\left(x^{(j-1)}, t_{j-1} \mid \mathbf{x}^{\prime}, t^{\prime}\right)$ is represented as a cubic $B$-spline series in the following manner

$$
\begin{gathered}
p\left(x^{(j-1)}, f_{j} \mid x^{\prime}, f^{\prime}\right)-\sum_{k_{i=1}}^{M_{1}} \cdots \sum_{k_{n}-1}^{w_{n}} \Gamma^{(j-1)}\left(k_{1}, \cdots, k_{n}\right) \\
\bigotimes_{i=1}^{n} B_{k_{i}}\left(x^{(j)}\right),
\end{gathered}
$$

where $M_{j}=$ number of grid points for the $\mathrm{i}^{\prime}$ th state variable $x_{i},\left\{\otimes_{i=1}^{n} B_{k_{1}}(\cdot)\right\}_{k-1}^{M_{i}}$ is a tensor product basis of cubic B-splines and $\left\{I^{\gamma}{ }^{\prime \prime}\left(k_{1}, \cdots, k_{n}\right)\right\}_{k_{r}}^{M_{r}}$ is the set of interpolation cofficients associated with time $t_{j}$, . It is assumed that cach set $\left\{B_{k_{i}}(\cdot)\right\}_{t_{i}+*}^{M_{*}}, i=I, \cdots, n$, is a basis of cubic $B$-splines associated with the knot sequence determined by the grid points for the i'th variable $x_{i}$. The tensor product B-spline is defined by

$$
\bigotimes_{i=1}^{n} B_{k_{i}}(x)=\prod_{i=1}^{n} B_{k_{i}}\left(x_{1}\right)
$$

The representation of $p\left(x^{(j-1)}, f_{j-1} \mid x^{\prime}, t^{\prime}\right)$ by B-splines makes it possible to retain high numerical accuracy even with a fairly coarse basic grid if $p\left(x^{(j-n)}, f_{j-1} \mid x^{\prime}, f^{\prime}\right)$ is not too singular. By substituting from Eq. (17) into Eq. (16), Eq. (19) is obtained

$$
\begin{array}{r}
p\left(x^{(j)}, l_{j} \mid x^{\prime}, t^{\prime}\right)=\sum_{k_{1}=1}^{M_{1}} \cdots \sum_{k_{n}=1}^{M_{n}} \Gamma^{j-1)}\left(k_{1}, \cdots, k_{n}\right) \\
\int \cdots \int p\left(x^{(i)}, t_{j} \mid x^{\left.(j-1), t_{j-1}\right)} \bigotimes_{j=1}^{n} B_{k_{i}}\left(x^{(j-1)}\right) \mathrm{d} x^{(j-1)} .\right.
\end{array}
$$

It is seen from Eq. (13) that since $m_{j}(\cdot)$ and $g_{i j}\left({ }^{\bullet}\right)$ are not functions of time $t$, the TPDs cannot depend on absolute time, but only on the time increment. Markov processes whose TPDs have this property, are called homogencous. It follow's that

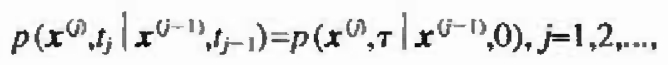

which holds for any $t_{j}-t_{j-1}=\tau \geq 0$.

From Eqs. (19) and (20) it is seen that for a fixed valuc of the time increment $\tau$, cach of the integrals on the right hand side of Eq. (19) need to be calculated only once, and can be stored for repeated use. That is, the following parameters are calculated initially and stored

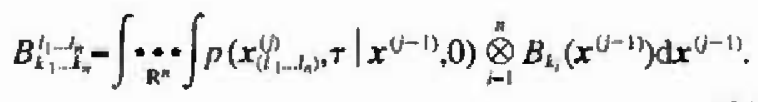

Here, the index $l_{i}, i=1, \ldots, n$, refers to grid point number $l_{i}$ for the state space variable $x_{i}$. It may be noted here that due to the properties of the TPD for small time increments $\tau$, the tensor $B_{k}^{i_{1}, t_{i}}$, has a strongly banded character with the clements decreasing rapidly away from the main diagonal $k_{1}=l_{1}, \ldots, k_{n}-l_{n}$. This has important implications for the efficiency of the computer program. Let

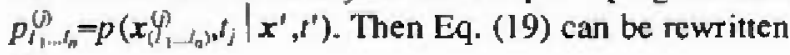
as

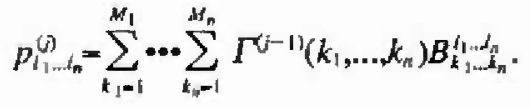

Having calculated the TPD $p\left(x^{(t)}, t_{j} \mid x^{\prime}, t^{\prime}\right)$ at the grid points by using Eq. (22), a spline interpolation is again carried out and a new set of interpolation coefficients $\left\{\Gamma^{0}\left(k_{1}, \ldots, k_{n}\right)\right\}_{t_{i}-1}^{k_{i}}$ are calculated. This provides an updated representation of the TPD for time step $j$, cf. Eq. (17). For each time step, the normalization condition Eq. (7) is checked. That is, if

$$
\begin{gathered}
\int \ldots \int p\left(x^{(j)}, t_{j} \mid x^{\prime}, t^{\prime}\right) \mathrm{d} x^{(j)}=\sum_{k_{1}=1}^{M_{1}} \ldots \sum_{k_{n}=1}^{M_{n}} I^{\left.w^{2}\right)}\left(k_{1}, \ldots, k_{n}\right) \\
\prod_{i=1}^{n} \int_{-\infty}^{\infty} B_{k_{1}}(x) \mathrm{d} x=q_{j}
\end{gathered}
$$


and $q_{j} \neq 1.0$ within the desired accuracy, then the follow. ing replacement is made to restorc the correct normalization.

$$
\Gamma^{(j)}\left(k_{1}, \cdots, k_{n}\right)^{\text {new }} \leftarrow q_{j}^{-1} \Gamma^{(j)}\left(k_{1}, \cdots, k_{n}\right)^{\text {nil }}
$$

This normalization check and replacement strategy contributes to producing a very stable and accurate numerical procedure.

\section{Examples}

The accuracy and power of the developed PIS procedure will be illustrated by application to specific case studies taken from two classes of dynamic models. Both models are described by Eq. (3) with $n-2$ and $m-3$. This implies a two-dimensional state space vector $X=\left(X_{1}, X_{2}\right)^{T}=(Z, Z)^{T}$. Fur ther, $m(\cdot)$ and $Q(\bullet)$ are such that $m_{1}\left(X_{1}, X_{2}\right)=X_{2}$ and $q_{1 j}(\bullet)=0$ for $j=1,2,3$. Assuming sufficient restrictions on $m(\bullet)$ and $Q(\bullet)$, cf. Refs. [6,7], $\boldsymbol{X}(t)$ becomes a Markow diffusion process. Invoking Eq. (13), it can be shown that, up to correction terms of order $\tau^{2}$, the associated TPD assumes the form

$$
p\left(x, \tau \mid x^{\prime}, 0\right)=\delta\left(x_{1}-x_{1}^{\prime}-x_{2}^{\prime} \tau\right) \cdot \tilde{p}\left(x_{2}, \tau \mid x^{\prime}, 0\right) .
$$

$\tilde{p}\left(x_{2}, \tau \mid x^{3}, 0\right)$ is given by the relation

$\bar{p}\left(x_{i}, \tau \mid \boldsymbol{x}^{\prime}, 0\right)-\frac{1}{\sqrt{2 \pi \beta\left(x^{\prime}\right) \tau}} \exp \left\{-\frac{\left(x_{2}-x_{2}{ }^{\prime}-m_{2}\left(x^{\prime}\right) \tau\right)^{2}}{2 \beta\left(x^{\prime}\right) \tau}\right\}$,

where

$$
\beta\left(x^{\prime}\right)=\sum_{j=1}^{3} q_{2 j}\left(x^{\prime}\right)^{2}
$$

By combining Eqs. (25) and (26), and applying the solution technique described in the previous section, the TPD $p\left(x, t \mid x^{\prime}, t^{\prime}\right)$ for largc $t-t^{\prime}$ can be calculated. By this, the time cvolution of the system when it starts from rest, for example, can be studied. The stationary PDF is obtained in the limit as $t-t^{\prime} \rightarrow \infty$. For application of the PIS method to other problems involving both two- and three-dimensional state space vectors, the reader may consult Refs. $[11-13,18,19]$.

\subsection{Example 1-The Caughey Oscillator}

Therc is a class of dynamic models for which there exist an analytical solution for the stationary joint PDF of $\boldsymbol{X}$. A member of this class may be called a Caughey oscillator, Ref. [20]. The generic equation of motion for this oscillator can be written as

$$
\ddot{Z}+\dot{Z} g(E)+h(Z)=\Gamma N(t) .
$$

$N(t)$ denotes a stationary, zero-mean Gaussian white noise satisfying $E[N(t) N(t+\tau)]=\delta(\tau)$, where $\delta(\cdot)$ denotes Dirac's delta function, $\boldsymbol{C}$ is a positive constant and $g(E)$ is a function of the total cnergy $E=E(Z, \dot{Z})$ given as follows

$$
E=\frac{1}{2} \dot{Z}^{2}+V(Z)
$$

where

$$
V(z)=\int_{0}^{\Sigma} h(s) \mathrm{d} s
$$

For this example $m_{2}(z, z)-z g[E(z, \dot{z})]-h(z)$, and we may choose $q_{21}=q_{22}=W_{1}-W_{2}=0 . \quad q_{23}=\Gamma$ and $\mathrm{d} W_{3}(t)-N(t) \mathrm{d} t$. The stationary, joint $\mathrm{PDF}$, denoted by $p_{s}(\bullet)$, is then determined by the relation, cf. Refs. $[20,21]$

$$
p_{s}(z, \bar{z})=C \exp \left\{-\frac{2}{T^{2}} \int_{0}^{F} g(s) \mathrm{d} s\right\}
$$

where $E^{\prime}=z^{2} / 2+V(z)$, and $C$ is a normalization constant to ensure a total probability equal to 1.0 .

For the illustration purposes in this paper, we have chosen the following special case of Eq. (28)

$$
\begin{aligned}
Z(t)+2 \xi \dot{Z}(t)\{1 & \left.+\varepsilon\left[\frac{1}{2} \dot{Z}^{2}(t)+\frac{1}{2} Z^{2}(t)+\frac{1}{4} \lambda Z^{4}(t)\right]^{1 / 2}\right\}+Z(t) \\
& +\lambda Z^{3}(t)=2 \sqrt{\xi} N(t)
\end{aligned}
$$

with parameters $\xi, \varepsilon$, and $\lambda$.

The stationary PDF only depends on the parametcrs $E$ and $\lambda$, and the numerical solution for the following set of parameter values has been calculated $(\varepsilon, \lambda)=(0,0)$ (Gaussian responsc), $(0,0.2)$ (Duffing oscillator) and $(0.5,0.1)$. The calculations were carried out with the same number of grid points on both axes in state space, aviz., 45. Since the resulting PDFs are actually independent of $\xi$, the value $\xi 0.1$ was chosen for the Gaussian and Duffing cases, while $\xi-0.5$ was adopted for the last case. The timc increments used were $\tau=0.0025 \mathrm{~s}, 0.001$ $\mathrm{s}$, and $0.02 \mathrm{~s}$, respectively. The total CPU time on a DEC station $3100^{\prime}$ was about 5 minutes for each case. In Figs:

'Certain commercial equipment, instruments, or materials are identified in this paper to specify adequately the experimental procedure. Such identification does not imply recommendation or endorsement by the National Instilute of Standaris and Technology, nor does it imply that the materials or equipmen identified are necessarily the best availahle for the purpose. 
1 and 2 are shown the marginal PDFs of the displacement response for the three case studies considicred, together with the corresponding analytical solutions. In Fig. 3 are given the corresponding analytical and numerical results for the mean upcrossing rate. It is seen that in all three cases the agreement between the numerical PIS and the analytical solution is very good over the whole range of probability levels given. In fact, the accuracy can be retained down to much lower probability levels $\left(\simeq 10^{-19}\right)$ at a moderate increase in computer time.

\subsection{Example 2-Parametric and External Excitation}

In this example, the response statistics of a nonlinear oscillator subjected to both cxternal and parametric random excitation will be illustrated by applying the methodology of the paper to two specific case studies.

The equation of motion of the oscillator is the following

$$
\ddot{Z}+2 \xi\left[1+\tilde{N}_{1}(t)\right] \dot{Z}+\gamma\left[Z^{2}+\frac{Z^{2}}{\omega_{0}^{2}} \mid \dot{Z}+\omega_{i}^{2}\left[1+\tilde{N}_{2}(t)\right] Z-\tilde{N}_{3}(t) .\right.
$$

Here $\xi, \gamma$, and $\omega_{0}$ are positive constants, $\bar{N}_{j}(t), j=1,2,3$, arc independent Gaussian white noises satisfying

$$
E\left[\tilde{N}_{j}(t) \tilde{N}_{j}(t+\tau)\right]=I_{j}^{*} \delta(\tau), j=1.2 .3 \text {, }
$$

where $J_{j}$ are positive constants. For this example it is found that $m_{2}(z, \dot{z})=-2 \xi \dot{z}-\gamma\left[z^{2}+\dot{z}^{2} / \omega_{1}^{2}\right]-\omega_{1}^{2} z$, $q_{21}(z, \dot{z})=-2 \xi \Gamma_{1} \cdot q_{22}(z, \dot{z})=-\omega_{13}^{2} \Gamma_{2}$ and $q_{23}(z, \dot{z})=\Gamma_{32}$

This model was studied by Dimentberg [22], who showed that when

$$
\omega_{1}^{2} I_{2}^{2}=4 \xi^{2} I_{1}^{2}
$$

a closed-form expression for the stationary joint PDF can be obtaincd. It is given as

$$
p_{s}\left(z, z^{2}\right)=C \frac{\exp \left\{-\mu\left(z^{2}+z^{2} / \omega_{0}^{2}\right)\right\}}{\left(\kappa+z^{2}+z^{2} / \omega_{0}^{2}\right)^{h-\alpha \mu}}
$$

where $\mathrm{C}$ is a normalization constant and

$$
\kappa=\frac{\Gamma_{3}^{2}}{\omega_{0}^{4} \Gamma_{2}^{2}}, \delta=\frac{2 \xi}{\omega_{11}^{2} \Gamma_{2}^{2}}+\frac{1}{2}, \mu=\frac{\gamma}{\omega_{0}^{2} \Gamma_{2}^{2}}
$$

By this, we have the opportunity to test the accuracy of the PIS method for this kind of dynamic model. The results of two particular cases will be presented.

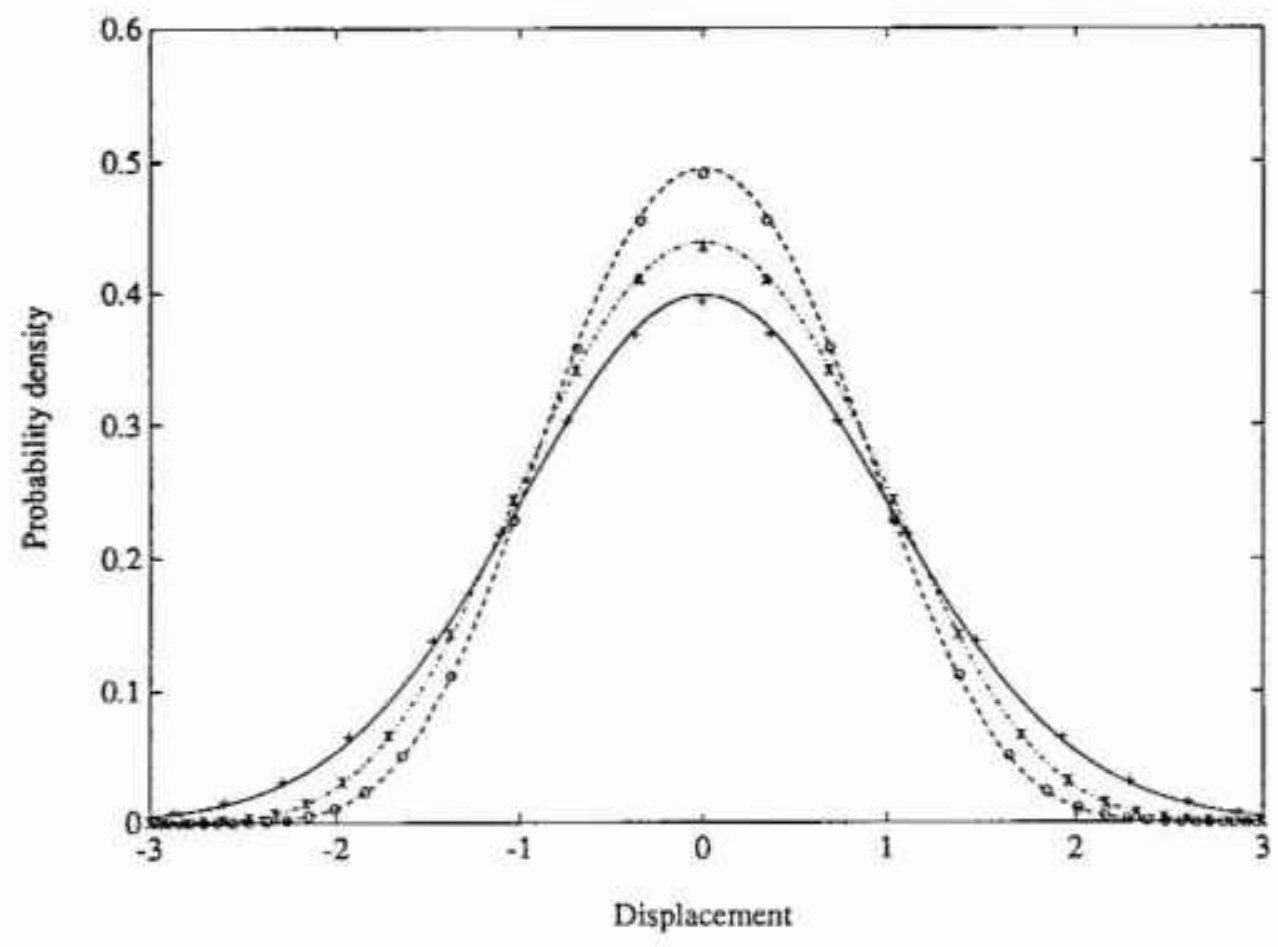

Fig. 1. Probability density function of displacement response for the Casughey oscillator in example 1. Analytical solutions: —, $E=0, \lambda=0 ; \ldots, \varepsilon=0, \lambda=0.2 ;----, \varepsilon=0.5, \lambda=0,1$. Numerical path inlcgal solution: $+, \epsilon=0, \lambda-0 ; \times, \varepsilon=0, \lambda=0.2 ; 0, t=0.5, \lambda=0.1$. 


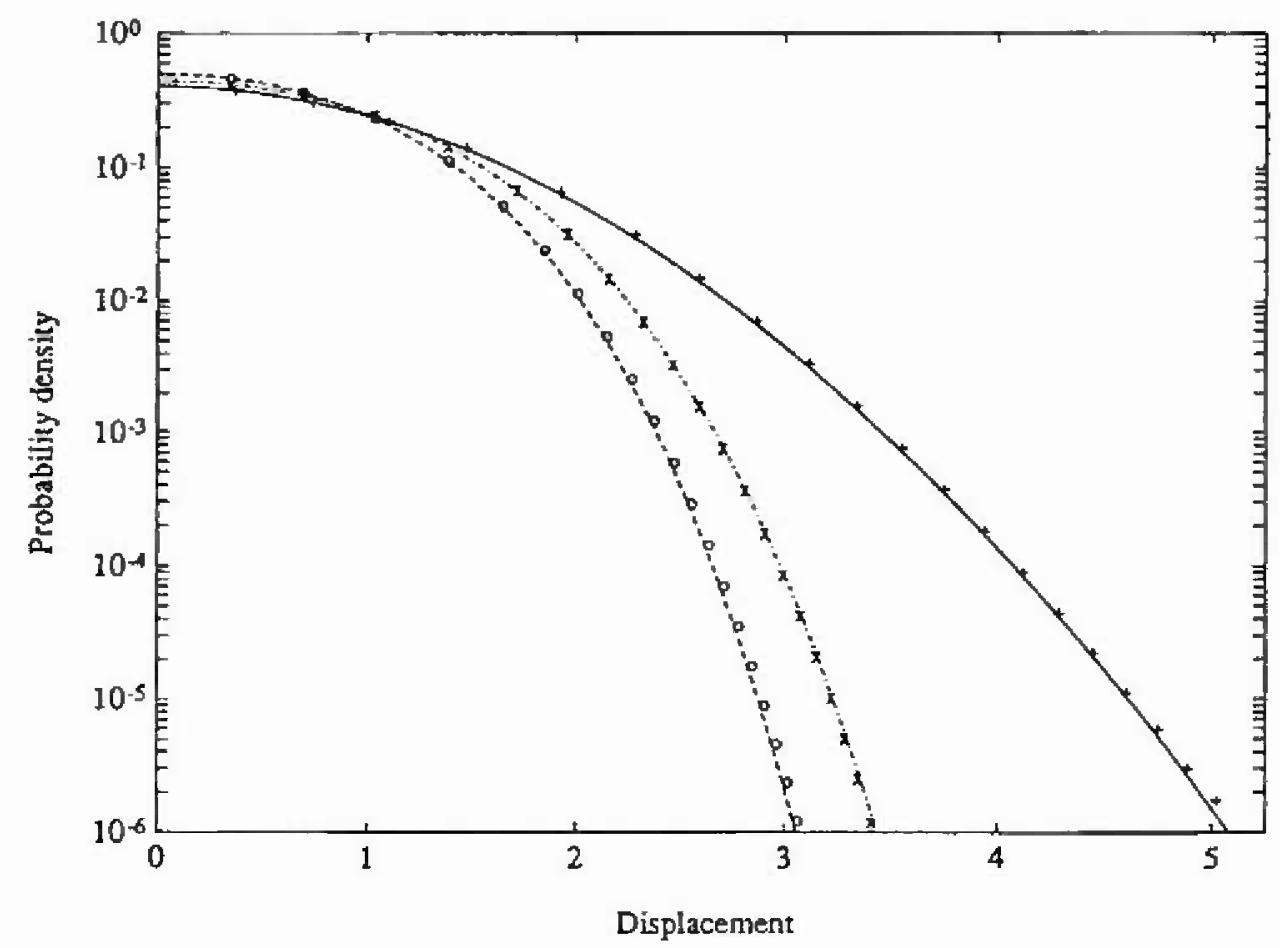

Fig. 2. Logarithmic plot of the protuability density funetion of displacement response for the Caughey oscillator in example 1. Key as in Fig. 1.

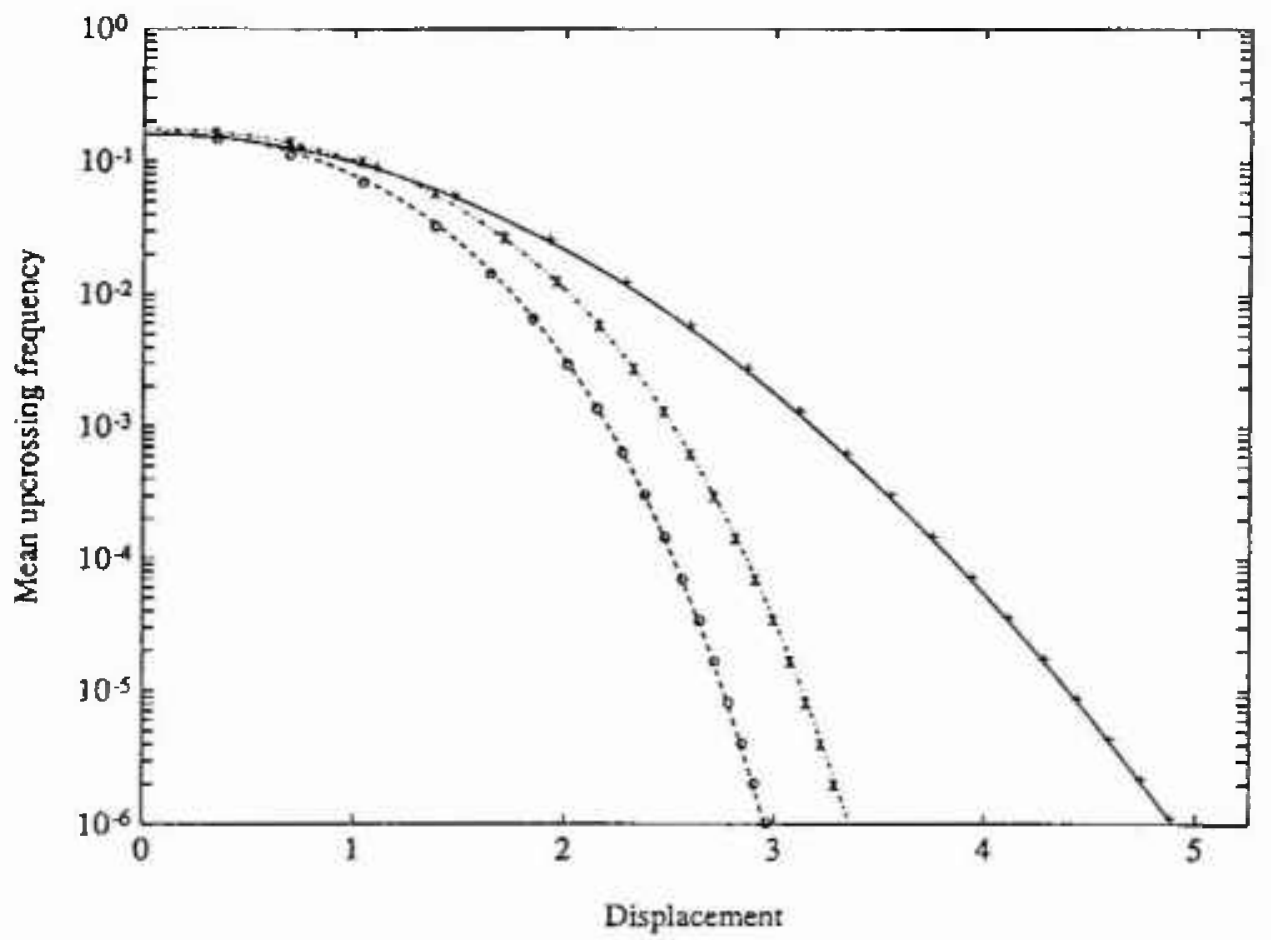

Fig. 3. Mean upcrossing rate of displacement response for the Canghey oscillator in example I. Key as in Fig. I. 
Case 1: Here the following parameter values were used. $\xi=0.1, \gamma=0.1, \omega_{0}=1.0, \Gamma_{1}^{2}=2.5, \Gamma_{2}^{2}=0.1, \Gamma_{3}^{2}=0.3$. For the numerical calculations a grid size of $49 \times 49$ points and a time increment $r=0.01 \mathrm{~s}$ was used. The total CPU time on a DEC 3100 work station was $3 \mathrm{~min}$ for the PIS calculation. The results for the analytical and numerical solutions are given in Figs. 4-6. In Figs. 4 and 5 are shown the marginal PDF of the displacement response and in Fig. 6 is shown the corresponding mean upcrossing rate.

Case 2: In this case the following sct of parameters were used. $\xi=0.1, \gamma=0.4, \quad \omega_{n}=1.0, \Gamma_{1}^{2}=5.0, \Gamma_{2}^{2}=0.2$, $\Gamma_{3}^{2}-0.3$. A grid size of $51 \times 51$ points together with a time increment $\mathrm{r}=0.01 \mathrm{~s}$ werc chosen. The CPU time was about the same as in the previous case. The same results as for Case 1 are presented in Figs. 4-6.

\section{Conclusions}

A numerical method for estimating the extreme response of nonlinear oscillators excited by white noisc, or filtered white noise, has been described. The example calculations presented show that the method gives very accurate estimates of the required joint PDF. In fact, for every example having analytical solution on which the method has been tested, complete agreement has been found with proper choice of grid size and time increment in the numerical solution procedure. In the present paper, of course, only a few cases can be given. Experience with the method indicates that two-dimensional problems can be solved routinely with high accuracy requiring a few minutes CPU time on a work station (DEC station 3100). The solution of three-dimensional problems requires more care in the sense that computer capacity starts to become an issue of importance. In such cases the CPU time easily runs into hours.

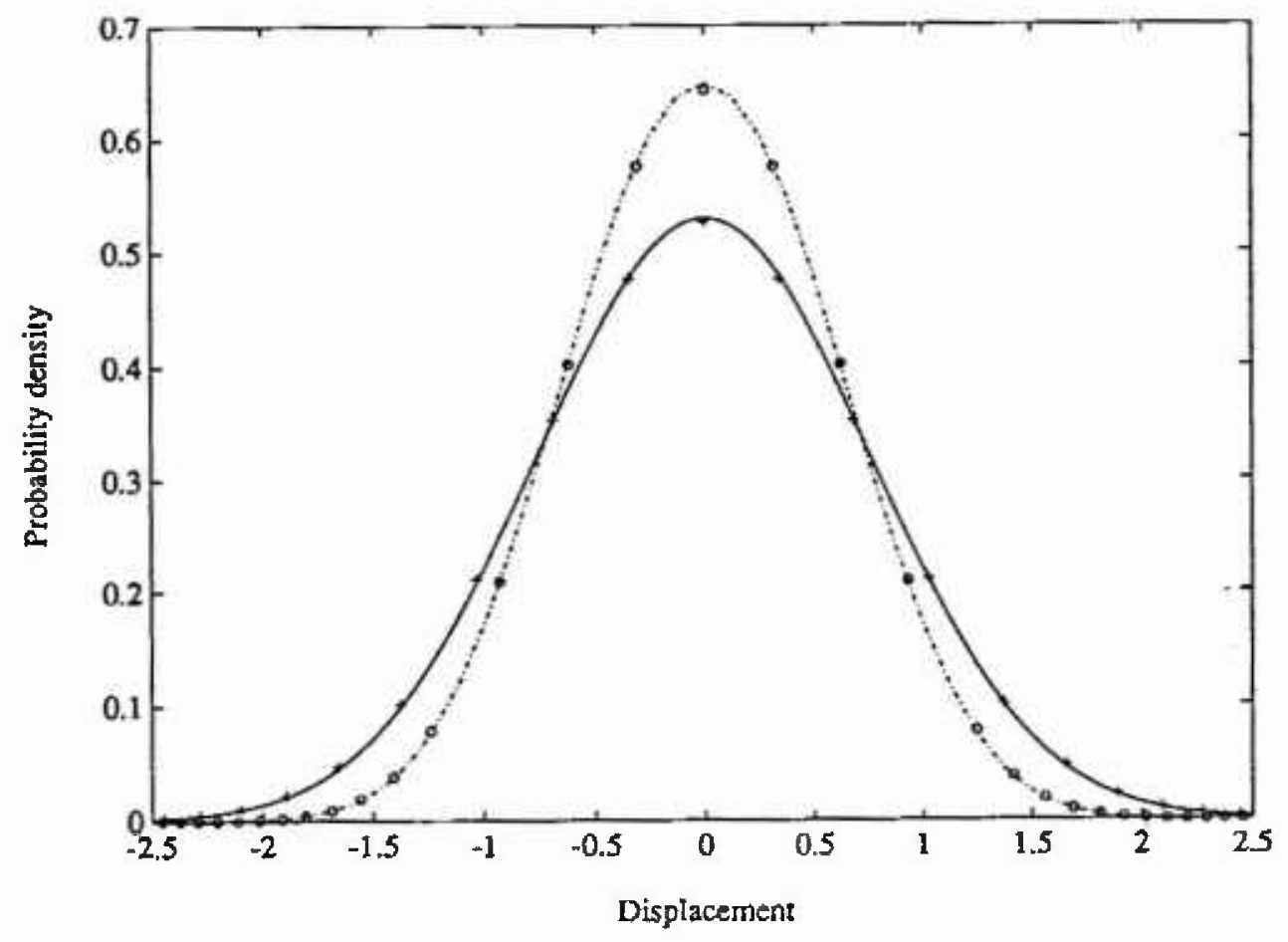

Hig. 4. Probability density function of displacement nesponse for the oscillanor in example 2, case 1 and 2. Analytical solutions: - _ case 1:-.- case 2. Numerical palh integral solution: +, case 1; 0. case 2 


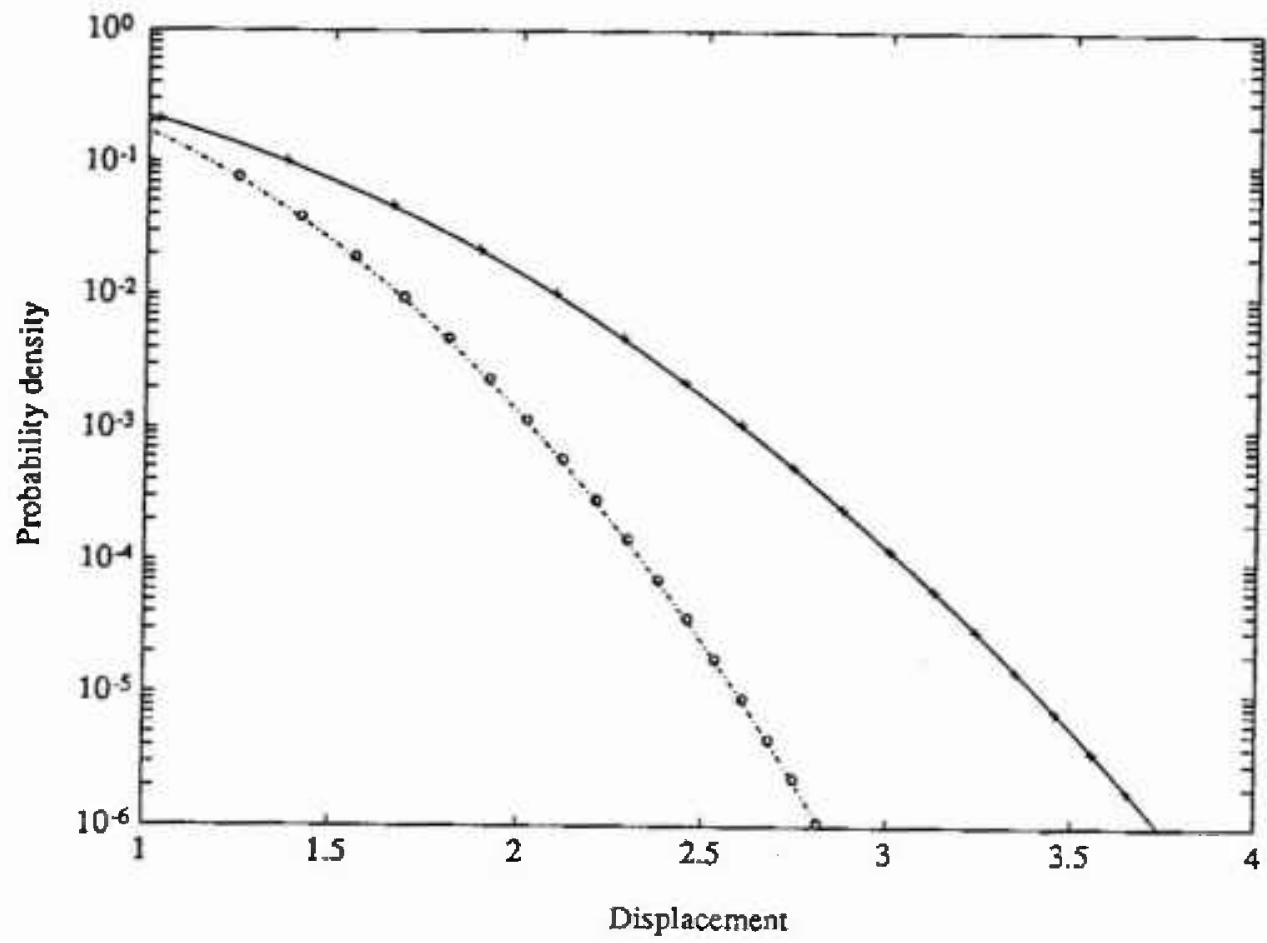

Fig. 5. Logarithmic plot of the probability density function of displacement response for the oscillator in example 2, case 1 and 2, Key as in Fig. 4.

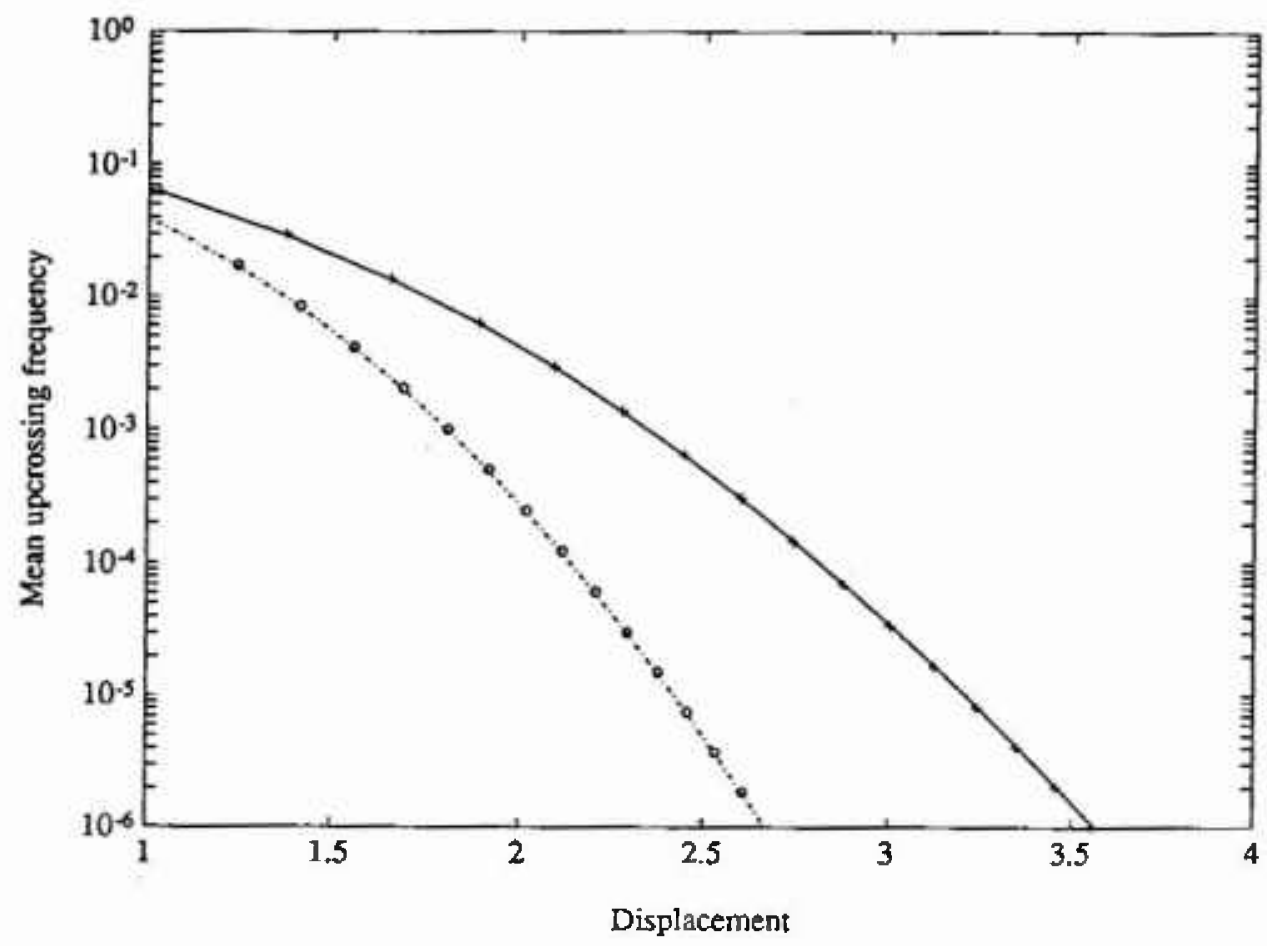

Fig. 6. Mcan upcrussing rate of displacement response for the oscillator in example 2, case 1 and 2. Kcy as in Fig. 4. 


\section{References}

[1] R. Y. Rubinstein, Simulation and the Monte Carlo Method. J. Wiley \& Sons, New York (1981).

[2] J. N. Franklin, Numerical simulation of stationary and non-stationary Gaussian random processes, SIAM Review 7 (1), 68-80 (1965).

[3] M. Shinozuka and C.-M. Jan, Digital Simulation of Random Processes and Its Applications, J. Sound Vibration 25 (1), 1 I I128 (1972).

[4] P. D. Spanos and M. D. Mignolet, ARMA Monte Carlo SimulaIion in Probabilistic Structural Analysis, Shock Vib. Diges 21, 3-14 (1989)

[5] F. Yamazaki and M. Shinozuka, Simulation of stochastic ficlds by statistical preconditioning, J. Struct. Eng., ASCE 116 (2), 268-287 (1990).

[6] E. Wong and B. Hajek. Stochastic Processes in Enginecring Systems, Springer-Verlag. New York (1985).

[7] H. Risken, The Fokker-Planck Equation, Second Edition, Springer-Verlag, Berlin (1989).

[8] M. F. Wheher and W. G. Wolfer, Numerical evaluation of path integral solutions to Folker-planck equations, Phys. Rev, A 27 (5), 2663-2670 (1983).

[9] T. Kapitaniak, Slochasic Response with Bifurcations to NonLinear Duffing"s Oscillator. J. Sound Vibration 102 (3). 440 441 (1985).

[10] A. Naess and J. M. Johnsen, Direct Numerical Simulation of the Response Statislics on Nonlinear Dynamic Systems, Proc, Scandinavian Forum for Stochastic Mechanics, Lund Institute of Technology, Lund, Sweden, August 1990.

[1I] A. Nacss and J. M. Johnsen. Response Statisics of Nonlinear Dynamic Systems by Path Integration. Proc. IUTAM Symposium on Nonlinear Stochanic Mechanics. Turin, Taly, July 1991.

[12] J, M. Johnsen and A. Naess, Time Variant Wave Drift Darnping and its Effect on the Response Statistics of Moored Offshore Sinuctures, Proc. Ist Int Conference on Orfshore and Polar Enginecring (ISOPE-91), Edinburgh, Augus 199I.

[13] A. Naess and J. M. Johnsen. The Path lntegral Solution Technicput applicd to the Random Vibration of Hystertic Systems. Proc. 1st Int. Conf. on Conputational Stochastic Mechanics. Corfu, Grece, September 1991, Computational Mechanics Publications, 1991.

[14] E. H. Vanmarcke, On the distribution of the first-passage lime for normal slationary random processes, J. Appl. Mech. 42, $215-220$ (1975).

[15] J. B. Roberts, First passage lime for the envelope of a randomly excired linear oscillator. J. Sound Vibration 46. 1-14 (1976).

[1.61 A. Naess. Approximate first-passage and extremes of narrowband Gaussian and non-Gaussian random vibrations, J. Sound Vibration 138, 365-380 (1990),

[17] C. de Boor, A Phectical Guide to Splines, Springer-Verlag, New Vork (1978).

[18] A. Naess and J. M. Johnsen, Response statistics of nonlinear, compliant offshore structures by the path integral solution method, Probabilistic Eng. Mech. 8. 91-106 (1993).

[191 A. Naess and B. K. Hegstad, Response statistics of van der Pol oscillators excited by white noise, Nonlinear Dynamics 5. 287297 (1994).

120] T. K. Caughey, Nonlinear theory of tundom vibrations, in Advances in Applied Mechanics, Academic Press, New York (1971).
[2.1] Y. K. Lin, Probabilistic Theory of Structural Dynamics. McGraw-Hill, New York (1967).

122] M. F. Dimentherg. An exact solvtion to a certain non-linear random vibration problem, Int, J, Non-Linear Mech. I7 (4). $23 i-236$ (1982).

About the author: Arvid Naess is Professor of Structural Engineering on the Faculiy of Civil Engineering of The Nonvegian Institute of Technology in Trondheim. Norway. 